\title{
Efectos de la privatización en la gestión financiera: un estudio de casos
}

\section{The effects of privatisation on the financial management: a case study}

\author{
Koldo Zabalza Miera / Jesús Matey de Antonio ${ }^{1}$ \\ Universidad del País Vasco
}

Recibido el 31 de marzo de 2010, aceptado el 5 de mayo de 2010

$\mathrm{N}^{\mathrm{o}}$ de clasificación JEL: M10

DOI: $10.5295 / \mathrm{cdg} .100214 \mathrm{kz}$

Reseña Bibliográfica: ZABALZA MIERA, K.; MATEY DE ANTONIO, J. (2010): "Efectos de la privatización en la gestión financiera: un estudio de casos"; Cuadernos de Gestión, Vol. 10. № 2, pp. 81-98. DOI: $10.5295 / \mathrm{cdg} .100214 \mathrm{kz}$

\section{Resumen:}

La privatización de una empresa conlleva un profundo proceso de reforma, que incluye la introducción de nuevas prácticas de gobierno. La revisión de trabajos teóricos y empíricos realizada nos permite confirmar que no existe un análisis sistemático de los cambios en la gestión que tienen lugar tras la privatización de una empresa pública. En este trabajo nos planteamos como objetivo principal explorar los cambios en la gestión financiera que se producen tras la privatización de una empresa pública y proponer factores relevantes en tres ámbitos de actuación de su gestión (estructura financiera, decisiones de inversión y planificación financiera). Para confirmar las proposiciones teóricas planteadas utilizamos el estudio de casos múltiple contemporáneo como metodología de investigación. En concreto investigamos los cambios experimentados en el área de gestión financiera de cuatro empresas privatizadas españolas, siguiendo un enfoque de análisis de corte longitudinal. Los resultados obtenidos permiten confirmar la mayoría de las proposiciones y concluir que las tres áreas consideradas son relevantes en el proceso de cambio en la gestión financiera de una empresa privatizada.

Palabras clave:

Privatización, cambios en la gestión, gestión financiera, estudio de casos.

\begin{abstract}
:
The privatisation of a firm entails a thoroughgoing process of reform that includes the introduction of new management practices. A review of the theoretical and empirical studies conducted allows us to confirm that a systematic analysis of the change in management that arise after a public company's privatisation does not exist. This study explores the changes in financial management that take place after a public company's privatisation and proposes relevant factors in three performance areas of financial management (financial structure, investment decision and financial planning). In order to confirm the theoretical propositions posed, we used contemporary multiple case studies as a research methodology. In particular, the changes experienced in the financial management area in four privatised Spanish companies were investigated along the lines of a longitudinal analysis. The results of this study show that most theoretical propositions are confirmed and conclude that the three areas considered are relevant to the process of change in a privatised firm's financial management.
\end{abstract}

Keywords:

Privatisation, changes in management, financial management, case study.

${ }^{1}$ Departamento de Economía Financiera II de la Universidad del País Vasco. Facultad de Ciencias Económicas y Empresariales. Plaza Oñati, 1. 20018 San Sebastián. koldo.zabalza@ehu.es. jesus.matey@ehu.es. 


\section{INTRODUCCIÓN}

Desde los años ochenta del siglo XX hemos asistido a una defensa de la iniciativa y la propiedad privada. Se ha extendido por todo el mundo un sentimiento anti-Estado o antisector público, que postula la minimización de la intervención estatal en la vida económica. Es decir, se ha optado por un modelo de desarrollo económico donde la propiedad privada y el mercado libre son sus actores principales, dejando un papel muy pequeño a la acción del Gobierno. En este contexto ideológico, ha resultado relativamente sencillo llevar a cabo los programas de privatización de empresas públicas en los diferentes países ${ }^{1}$.

La importancia económica del proceso privatizador y su alcance, tanto por el número de países como por la intensidad con que cada país ha privatizado su sector público empresarial, ha impulsado fuertemente las investigaciones en este campo en los últimos años (Boubakri y Hamza, 2007; Boubakri et al., 2005; D’Souza et al., 2005; Megginson et al., 2004; Parker y Kirkpatrick, 2005). Además, los investigadores disponen ahora de empresas con un horizonte temporal suficientemente amplio tras su privatización para poder realizar estudios más relevantes.

La mayoría de los estudios empíricos realizados sobre la privatización se han centrado en analizar los resultados de la empresa, para valorar su eficiencia, con dos enfoques distintos: por un lado, estudios comparativos de empresas públicas y privadas que trabajan simultáneamente en el mercado, en un momento determinado de tiempo, y con un análisis de corte transversal (Foreman-Peck y Waterson, 1985; Millward y Ward, 1987; Pryke, 1982; Atkinson y Halvorsen, 1986; Bruggink, 1982; Di Lorenzo y Robinson, 1982; Dewenter y Malatesta, 2000; Laurin y Bozec, 2001; Millward y Parker, 1983; Tian, 2000); y por otro, estudios de casos antes y después de una privatización, analizando la evolución de los resultados de una misma empresa en sus diferentes etapas de titularidad pública o privada, y con un análisis de corte longitudinal (Burns y Weyman-Jones, 1994; Eckel et al., 1997; Foreman-Peck, 1989; Lynk, 1993; Martin y Parker, 1995; Boardman et al., 2003; Boubakri y Cosset, 1998; D’Souza y Megginson, 1999; Megginson et al., 1994).

Sin embargo, quizá no sea suficiente preguntarse si el cambio de titularidad provoca un aumento de la eficiencia. La privatización de una empresa conlleva un profundo proceso de reforma, que incluye la introducción de nuevas prácticas de gobierno (Estrin, 2002). El análisis de los cambios internos que tienen lugar tras la privatización permitirá entender mejor la relación entre la privatización y la eficiencia, y motivará que surjan nuevas investigaciones en este campo. García Álvarez y Mariz (2003, p. 1094) señalan que son muchos los trabajos empíricos que han tratado de comprobar si la privatización conlleva un incremento de eficiencia, pero que, sin embargo, los trabajos teóricos y empíricos relacionados

\footnotetext{
${ }^{1}$ La actual crisis económica ha tenido un doble efecto. En un primer momento ha provocado la intervención de diferentes Gobiernos en el sector privado para evitar el riesgo de colapso económico y financiero, mediante procesos de nacionalización en distinto grado (EEUU, Reino Unido, Bélgica, Holanda, Austria, Alemania o Irlanda). Según el barómetro que elabora la Fundación italiana Eni Enrico Mattei, 2008 fue el primer año desde 1981 en que los gobiernos a nivel mundial adquirieron más activos al sector público que de los que se deshicieron a través de programas de privatización. Sin embargo, son varios los países que están recurriendo a la privatización de sus participaciones accionariales (Grecia, Reino Unido o EEUU) para tratar de reducir el déficit y la deuda pública provocados por la crisis.
} 
con la identificación de los factores concretos que conllevan tal mejora de eficiencia son prácticamente inexistentes ${ }^{2}$.

En este contexto, y con el ánimo de contribuir a cubrir en alguna medida este gap, realizamos el presente trabajo de investigación centrándonos en el estudio de los cambios en la Dirección Financiera que se producen en las empresas públicas que son privatizadas.

Estructuramos este trabajo de la siguiente forma. Comenzamos con la exposición de los objetivos (tanto principal como secundarios) y de las proposiciones teóricas sobre los factores de cambio en la Dirección Financiera en un proceso de privatización. A continuación, explicamos y justificamos el uso de la metodología de investigación basada en el estudio de casos. En el siguiente apartado realizamos el análisis comparativo de los casos investigados en base a sus comportamientos factoriales. Y finalizamos con la exposición de las principales conclusiones extraídas de la investigación y una breve referencia a las limitaciones del estudio.

\section{OBJETIVOS Y PROPOSICIONES TEÓRICAS}

El objetivo principal de este trabajo es explorar los cambios en la Dirección Financiera que se producen tras la privatización de una empresa pública. Para ello, formulamos un conjunto de proposiciones teóricas sobre los factores relevantes para explicar esos cambios, valoramos el grado de coincidencia entre las proposiciones teóricas de partida y los comportamientos desarrollados por las empresas analizadas y determinamos la existencia o no de un patrón de comportamiento común entre ellas.

Los factores relevantes que sustentan las seis proposiciones teóricas y sirven de base para el desarrollo del estudio empírico se construyen tras una revisión bibliográfica y un proceso de consulta a expertos en Dirección Financiera.

Las proposiciones aparecen agrupadas en tres áreas: estructura financiera, decisiones de inversión y planificación financiera.

\subsection{Estructura financiera}

Las empresas públicas se han caracterizado por tener un gran endeudamiento que aumenta su riesgo financiero y provoca que la rentabilidad de la empresa disminuya a medida que aumenta la deuda, ya que la rentabilidad de las nuevas inversiones no supera el coste de la financiación ajena (Gómez y García, 2004, p. 870). En esta línea, Megginson (2004, p. 406) considera que el apalancamiento financiero ${ }^{3}$ disminuye tras la privatización.

Además, las empresas privatizadas mediante una Oferta Pública de Venta (OPV) obtienen más facilidades para captar recursos financieros en los mercados de capitales y así reducir la deuda (Bradley et al., 1984). Como señala Durán (2004, p. 843) la privatización de empresas "puede ampliar no sólo el mercado doméstico sino las opciones de finan-

\footnotetext{
${ }^{2}$ No obstante, hay algunos autores, como Zahra et al. (2000), Rondinelli (2004) y Cuervo (2004) que han tratado de explicar con sus modelos teóricos los cambios producidos en la empresa tras someterse a un proceso de privatización.

${ }^{3}$ Definido como deuda total entre activo total.
} 
ciación de carteras internacionales, si inversores extranjeros adquieren acciones de la empresa privatizada o si ésta vende sus títulos en los mercados internacionales". De este modo, la empresa puede tanto vender sus acciones en mercados exteriores organizados como endeudarse en divisas.

González-Páramo y Hernández de Cos (2004, p. 663) introducen otro argumento para justificar un menor endeudamiento tras la privatización, y es que el tipo de interés implícito de la deuda se eleva al no contar con la garantía del Estado.

Arcas y Ruiz (1999, p. 32), en cuanto al caso de Repsol, demuestran que los efectos de cada una de las OPV analizadas son distintos. Así, en los dos ejercicios posteriores a la OPV de 1989, la salida a Bolsa de Repsol no supone una reducción del endeudamiento, sino que aumenta debido a la necesidad de financiar el mayor volumen de inversiones. Por el contrario, tras la OPV de 1993 el efecto sobre el endeudamiento es el esperado.

Por otra parte, con la privatización aumentará la preocupación de la empresa por su riesgo financiero, al no tener al Estado detrás para cubrirle. En consecuencia, aumentará el empleo de mecanismos de cobertura del riesgo financiero como los futuros, los contratos a plazo (forward), los swaps o las opciones. Además, como consecuencia de la multinacionalización de las fuentes del capital financiero, la empresa debe cubrirse del riesgo de cambio con permutas o swaps de divisas (Durán, 2004, p. 852).

En definitiva, las aportaciones de todos estos autores nos conducen al establecimiento de las siguientes proposiciones teóricas:

* P1: Con la privatización, la empresa reduce su nivel de endeudamiento.

P2: La privatización provocará un aumento de la utilización de mecanismos de cobertura del riesgo financiero (futuros, contratos a plazo, swaps, opciones).

\subsection{Decisiones de inversión}

Respecto al nivel de inversión, hay que señalar que no hay unanimidad entre los distintos autores. Existen argumentos para justificar tanto un aumento como una disminución de la inversión tras la privatización. Así, Boycko et al. (1996) consideran que las empresas públicas invierten más que las privadas, sobre todo porque cuentan con financiación pública para realizar esas inversiones.

Sin embargo, Megginson et al. (1994) defienden que tras la privatización aumenta la inversión en la empresa. Primero, porque tras una OPV inicial tiene acceso a nuevos recursos financieros en el mercado de capitales, aumentando sus posibilidades de inversión. Y segundo, porque si la privatización va acompañada de medidas de liberalización del sector en cuestión, la empresa deberá hacer más inversiones para mantener su nivel competitivo. También Iranzo (2004, p. 817) considera que cuando las empresas son privatizadas se produce un aumento del esfuerzo inversor. Además, en las empresas públicas los directivos pueden considerar que los beneficios de las nuevas inversiones pueden ser expropiados y que difícilmente obtendrán compensaciones por los mismos. Con la privatización tendrán más incentivos para llevar a cabo nuevas inversiones, aumentando el grado de riesgo que están dispuestos a asumir ya que esperan que serán recompensados con parte de los beneficios obtenidos (Laffont y Tirole, 1993). 
Arcas y Ruiz, en su trabajo sobre los efectos de la privatización de empresas sobre el comportamiento económico y financiero (1999, p. 33) señalan que "las inversiones no aumentan tras la privatización, sino que se anticipan a ella. Las mayores inversiones se realizan con anterioridad a la privatización para adaptar la empresa a la nueva estructura accionarial y a la liberalización del sector".

Respecto a la autorización de proyectos, en la empresa pública, la decisión de inversión tiende a reservarse a la dirección general y se ponen límites al tamaño de los proyectos que pueden ser autorizados por otros niveles jerárquicos. Creemos que tras la privatización se da una mayor descentralización de las decisiones de inversión de capital, independientemente de cuáles sean los procedimientos formales aplicados. Además, en ocasiones, las inversiones deben ser aprobadas por las autoridades de control siguiendo criterios políticos (Aharoni, 1981; Shleifer y Vishny, 1994).

En relación con el tipo de inversión llevada a cabo, consideramos que la empresa pública desarrolla sobre todo inversiones de renovación o reemplazo, orientadas a mantener la capacidad productiva. Después de la privatización, las inversiones son principalmente de modernización, orientadas a la introducción de la innovación en la empresa, tanto en los productos como en los procesos productivos.

García y Mariz (2003, p. 1087) consideran que las empresas públicas invierten poco en nuevas tecnologías porque temen que ello pudiera suponer despidos de trabajadores, que tratan de evitar por presiones políticas. Sin embargo, la empresa privada necesita renovar constantemente su tecnología si quiere continuar siendo competitiva en el mercado.

En definitiva, se podrían establecer las siguientes proposiciones:

* P3: La privatización produce un aumento de la inversión de la empresa.

* P4: El tamaño de los proyectos que no requieren la autorización de la dirección general aumenta tras la privatización, produciéndose una mayor descentralización en las decisiones de inversión de capital.

P5: Tras la privatización, aumentan las inversiones de modernización, en mayor medida que las de renovación o reemplazo.

\subsection{Planificación financiera}

El plan financiero describe la estrategia financiera de la empresa y proyecta sus futuras consecuencias, establece objetivos financieros y es un punto de referencia para evaluar el comportamiento posterior.

La complejidad e importancia de los planes financieros varía enormemente de una empresa a otra. Si consideramos que las empresas públicas pueden financiarse de forma regular, por ejemplo a través de la emisión de obligaciones, y que contemplan un abanico de oportunidades de inversión limitadas, esto permite que sus planes financieros sean rutinarios y no excesivamente complejos (Brealey y Myers, 1995, p. 856).

Por lo tanto, parece que podemos establecer la siguiente proposición teórica:

* P6: Tras la privatización ha aumentado la complejidad e importancia de los planes financieros desarrollados por la empresa. 


\section{METODOLOGÍA}

Para confirmar las proposiciones teóricas planteadas se utiliza el estudio de casos múltiple contemporáneo como metodología de investigación (Yin, 1989, 1993, 1994, 1998; Eisenhardt, 1989, 1991; Eisenhardt and Graebner, 2007; Siggelkow, 2007) con un enfoque científico esencialmente inductivo y, parcialmente, deductivo (Yin, 1994).

El enfoque científico del estudio empírico es: fundamentalmente, de inducción analítica a través de la lógica de la réplica (generalización analítica) con la que se pretende generalizar desde la experiencia de los casos particulares; y parcialmente deductivo, ya que se pueden generar procesos deductivos en la medida que se parte de proposiciones teóricas obtenidas previamente de la revisión de teorías, que son comprobadas empíricamente.

En este estudio investigamos los cambios experimentados en el área de Dirección Financiera de algunas empresas privatizadas españolas, siguiendo un enfoque de análisis de corte longitudinal. Analizamos la evolución de la empresa, en aquellos aspectos que constituyen factores relevantes en el proceso, en sus diferentes etapas de titularidad pública y privada. En el Anexo 1 presentamos la ficha técnica del estudio empírico, destacando los principales aspectos metodológicos de la presente investigación.

La unidad de análisis se concreta en empresas españolas con procesos de privatización consolidados. Así, analizamos los casos de: ENDESA, IBERIA, INDRA y TELEFÓNICA. La selección realizada no constituye una muestra representativa de una población, con capacidad de generalización estadística, sino una muestra teórica y lógica con capacidad de generalización analítica (Yin, 1994). Se ha tratado de escoger aquellos casos que ofrecían una mayor capacidad explicativa y una disposición adecuada por parte de sus directivos para afrontar los compromisos de la investigación. En el Anexo 2, se recogen diferentes aspectos de las cuatro empresas analizadas que permiten elaborar un perfil básico de cada una de ellas.

En el proceso de recogida de información se ha recurrido a diversas técnicas, tales como la revisión de documentos, el envío de cuestionarios para su cumplimentación y las entrevistas en profundidad a varios directivos de las empresas privatizadas seleccionadas. De este modo, podemos contrastar los diferentes tipos de datos recogidos en el proceso cumpliendo el principio de triangulación que garantiza la validez interna y constructiva de la investigación (Yin, 1989, p. 29).

Finalmente, se han seguido dos estrategias genéricas de análisis (Arias, 2003): en primer lugar, desarrollamos la descripción de cada caso individualizado, dando como resultado cuatro casos descriptivos; y en segundo lugar, las proposiciones obtenidas de la revisión teórica son contrastadas con la información obtenida, a través de la búsqueda de un patrón de comportamiento común (análisis pattern matching) y, puntualmente, a través de otra modalidad, la creación de explicación (explanation building $)^{4}$.

\footnotetext{
${ }^{4}$ La búsqueda de un patrón de comportamiento común (análisis pattern matching) es un procedimiento analítico que consiste en comparar un patrón obtenido empíricamente con otro preestablecido basado en predicciones teóricas. Si los resultados coinciden aumenta su validez interna (Chiva, 2001, p. 123). Es una técnica útil para relacionar los datos con las proposiciones y reflejar una situación donde diferentes partes de la información extraída de un caso se pueden relacionar con algunas proposiciones teóricas (Villarreal, 2007).

La creación de explicación (explanation building) consiste en aportar aclaraciones detalladas y razonadas de todas las afirmaciones y datos sobre el fenómeno (Chiva, 2001, p. 123). Esta técnica es de naturaleza iterativa, por lo que la explicación final es el resultado del seguimiento de una serie de etapas, pudiendo diferir de los planteamientos
} 
El rigor y la calidad del estudio empírico se basa en la validez (constructiva, interna y externa) y en la fiabilidad del mismo (Yin, 1994, 1998; Gibbert et al, 2008). En el Anexo 3 se exponen las distintas pruebas de evaluación del rigor y la calidad de esta investigación con las correspondientes tácticas y fases en las que tienen lugar.

\section{ANÁLISIS COMPARATIVO DE LOS CASOS INVESTIGADOS}

Las diferentes proposiciones han sido valoradas en cuanto a su grado de relevancia en la explicación del fenómeno siguiendo una escala Likert. El análisis de estos valores ha llevado a los resultados recogidos en la Tabla 1.

Tabla 1

Resumen de los principales resultados obtenidos en relación con las proposiciones planteadas en los cuatro casos analizados a través del análisis pattern-matching

\begin{tabular}{|l|l|l|l|l|l|l|}
\hline \multicolumn{2}{|c|}{ Áreas y proposiciones } & $\begin{array}{c}\text { ENDESA } \\
\text { (Caso 1) }\end{array}$ & \multicolumn{1}{|c|}{$\begin{array}{c}\text { IBERIA } \\
\text { (Caso 2) }\end{array}$} & $\begin{array}{c}\text { INDRA } \\
\text { (Caso 3) }\end{array}$ & $\begin{array}{l}\text { TELEFÓNICA } \\
\text { (Caso 4) }\end{array}$ & \multicolumn{1}{c|}{$\begin{array}{c}\text { Grado de } \\
\text { Confirmación }\end{array}$} \\
\hline $\begin{array}{l}\text { ESTRUCTURA } \\
\text { FINANCIERA }\end{array}$ & P1. & $\begin{array}{l}\text { No } \\
\text { confirmado }\end{array}$ & $\begin{array}{l}\text { No } \\
\text { confirmado }\end{array}$ & $\begin{array}{l}\text { No } \\
\text { confirmado }\end{array}$ & $\begin{array}{l}\text { No confirmado } \\
\text { CONFIRMACIÓN } \\
\text { UNÁNIME }\end{array}$ \\
\cline { 2 - 7 } & P2. & Confirmado & Confirmado & Confirmado & Confirmado & $\begin{array}{l}\text { CONFIRMACIÓN } \\
\text { UNÁNIME }\end{array}$ \\
\hline $\begin{array}{l}\text { DECISIONES DE } \\
\text { INVERSIÓN }\end{array}$ & P3. & Confirmado & Confirmado & Confirmado & No confirmado & $\begin{array}{l}\text { Múltiples } \\
\text { posiciones } \\
\text { con tendencia } \\
\text { confirmatoria }\end{array}$ \\
\cline { 2 - 7 } & P4. & Confirmado & Confirmado & $\begin{array}{l}\text { No } \\
\text { confirmado }\end{array}$ & Confirmado & $\begin{array}{l}\text { Múltiples } \\
\text { posiciones } \\
\text { con tendencia } \\
\text { confirmatoria }\end{array}$ \\
\cline { 2 - 7 }
\end{tabular}

Fuente: Elaboración propia

A continuación, incidimos en el análisis comparativo entre los casos estudiados respecto a cada uno de los factores clave destacados en las correspondientes proposiciones. La coincidencia o similitud de factores explicativos daría lugar, de forma exploratoria, a un

recogidos inicialmente en el patrón. Esta técnica lleva a analizar la evidencia del estudio de casos, revisar las proposiciones teóricas y volver a estudiar la evidencia desde una nueva perspectiva en este círculo iterativo (Sosa, 2006, p. 165). 
patrón de comportamiento factorial entre las empresas analizadas. Para ello, fijándonos en cada uno de los factores clave implícitos en su correspondiente proposición, comparamos la evaluación obtenida a través de la escala Likert en cada uno de los casos estudiados.

La representación gráfica de los perfiles factoriales nos permite establecer una radiografía del comportamiento factorial de las empresas estudiadas. Así, en el Gráfico 1 presentamos los perfiles de las cuatro empresas investigadas con la intención de mostrar gráficamente las diferencias o similitudes existentes en el patrón factorial de comportamiento de las empresas objeto de estudio.

\section{Gráfico 1}

\section{Perfiles gráficos de los factores estudiados del área Financiera en las cuatro empresas analizadas (escala Likert)}

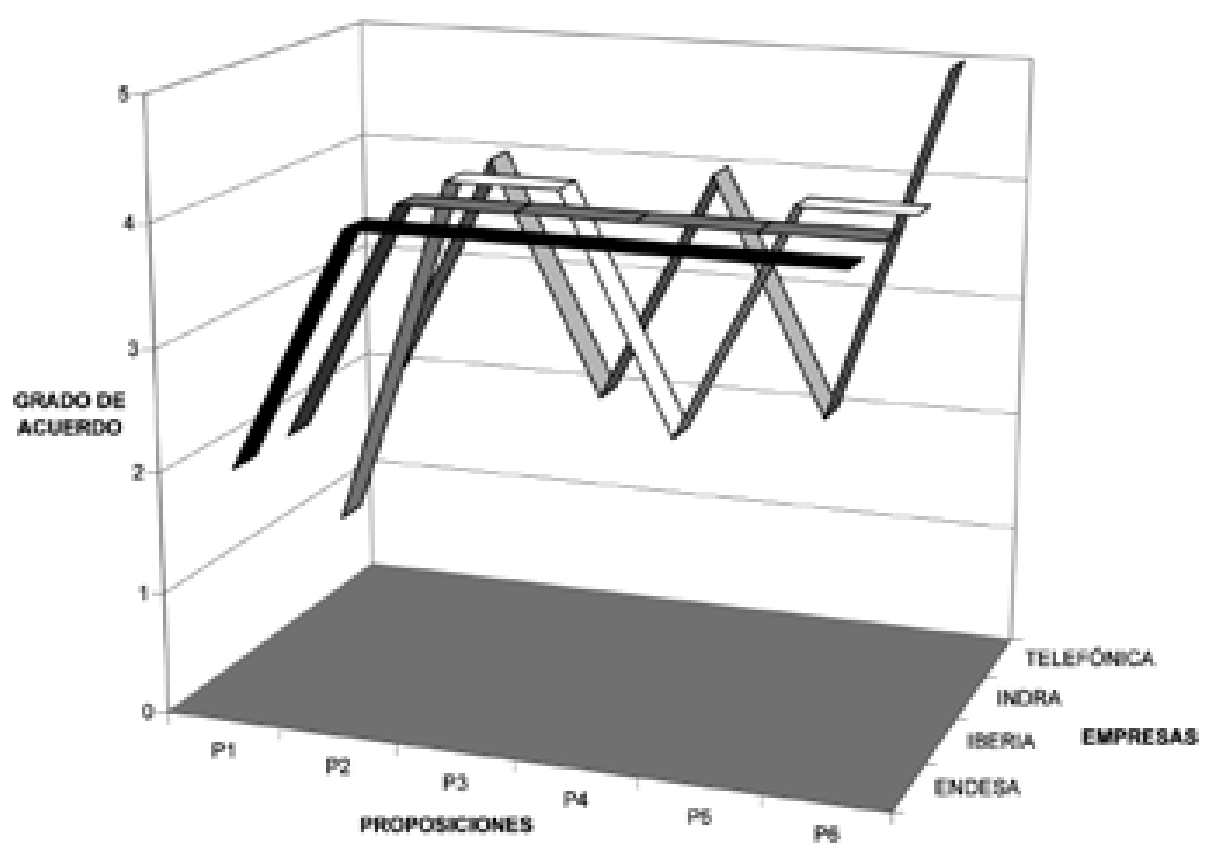

Fuente: Elaboración propia

Parece inducirse un patrón factorial con todos los factores absolutamente coincidentes entre Endesa (caso 1) e Iberia (caso 2), cinco de ellos confirmados y uno no confirmado. Y prácticamente también se produce la plena coincidencia con Indra (caso 3), con la única salvedad de la proposición P4. Por otro lado, dos de las seis proposiciones han alcanzado un nivel confirmatorio (posiciones 4 y 5 de la escala Likert) en todos los casos, y la proposición P1 ha sido rechazada en todos los casos. Las otras dos proposiciones han resultado confirmadas en tres de los cuatro casos. 


\section{CONCLUSIONES}

Las seis proposiciones teóricas de partida que han sido formuladas en los diferentes ámbitos considerados, recogen potenciales factores relevantes en el proceso de cambio en la Dirección Financiera de una empresa privatizada.

La mayoría de las proposiciones teóricas son confirmadas o tienden a serlo según la evidencia mostrada por las empresas de la muestra.

Existe cierta similitud entre algunos perfiles factoriales de las empresas estudiadas. En cualquier caso, la existencia de disparidades entre algunos de los casos impide realizar conclusiones rotundas sobre la posibilidad de confirmación de patrones.

El análisis comparativo de los comportamientos de las distintas empresas analizadas siguiendo la estructura construida a través de cada una de las áreas señaladas permite concluir que todas las áreas son relevantes en el proceso de cambio en la Dirección Financiera de la empresa privatizada.

El trabajo realizado sugiere una serie de implicaciones tanto para la teoría como para la práctica. Respecto de las implicaciones teóricas, el marco teórico que definimos ofrece una visión integrada de factores clave de la gestión financiera que deben ser estudiados en los procesos de privatización. En relación con las implicaciones prácticas, este estudio permite a los directivos de las empresas privatizadas y a la sociedad en general conocer las consecuencias que han tenido las políticas de privatización en la gestión financiera de la empresa. Quizás lo más interesante del estudio es que pone de manifiesto los cambios internos, posibilitando que otras empresas públicas puedan aplicarlos sin esperar a su privatización.

Por otra parte, debemos señalar dos limitaciones de la investigación. En primer lugar, no es posible la realización de ningún tipo de inferencia de naturaleza estadística con la intención de generalizar los resultados. Sin embargo, no debemos olvidar que un estudio de casos permite la generalización de naturaleza analítica, es decir, la ampliación de los planteamientos teóricos existentes en función del análisis de la evidencia obtenida a través de la lógica de la construcción de una explicación. Además, si tenemos en cuenta los motivos y los objetivos de esta investigación, estas limitaciones son claramente superadas por las ventajas que el estudio de casos ha aportado a este trabajo. Y en segundo lugar, el estudio se limita al análisis del cambio en el comportamiento antes y después de la privatización, sin controlar el posible impacto de otros factores. En particular, no se tiene en cuenta el efecto del ciclo económico, la evolución del marco competitivo u otros factores de carácter sectorial que pueden resultar comunes a todas las empresas, privatizadas o no, y que pueden influir de manera notable en los cambios producidos en la gestión.

Para finalizar, debemos señalar que la integración teórica debería llevar aparejada una depuración factorial tras el estudio empírico. Sin embargo, los resultados obtenidos en nuestro estudio apenas permiten descartes significativos. Parece que estudios posteriores deberían poner especial atención al tipo de consideración que cabría otorgar al factor recogido en la proposición P1, pudiendo quizás enunciarse en un sentido distinto al seguido en este estudio. Además, estimamos que quizá la agrupación factorial no ha sido excesivamente amplia y se requiera de un mayor desglose para su confirmación. En futuras investigaciones sería recomendable realizar un mayor desglose.

De la misma forma, otras investigaciones podrían considerar las siguientes cuestiones: aumentar el tamaño de la muestra, utilizando otras técnicas de investigación de carácter 
cuantitativo, que permitan una generalización estadística; incorporar diferentes metodologías de investigación, como el empleo de técnicas de consenso de expertos (Método Delphi), para reducir la subjetividad de la discriminación de factores que hemos realizado al elaborar las proposiciones teóricas; realizar el estudio en una muestra de control de empresas privadas y públicas que pertenezcan al mismo sector de actividad y con una dimensión similar, para poder comparar con los resultados de nuestra muestra de empresas privatizadas; y/o replicar el estudio en otros países con un grado de desarrollo y características políticas, económicas y sociales similares al nuestro, para que los resultados sean comparables. 


\section{ANEXO}

\section{Anexo 1 : Ficha técnica del estudio empírico}

\begin{tabular}{|c|c|}
\hline $\begin{array}{l}\text { Propósito de } \\
\text { investigación }\end{array}$ & $\begin{array}{l}\text { Analizar los cambios en la gestión de las empresas privatizadas. } \\
\text { Valorar el grado de coincidencia entre los comportamientos desarrollados } \\
\text { por las empresas privatizadas analizadas y las proposiciones teóricas de } \\
\text { explicación del fenómeno. } \\
\text { Perfilar un patrón de comportamiento común o similar (en el caso de que lo } \\
\text { haya) entre las empresas de la muestra. }\end{array}$ \\
\hline $\begin{array}{l}\text { Metodología de } \\
\text { investigación }\end{array}$ & $\begin{array}{l}\text { Estudio de casos contemporáneo múltiple de carácter holístico (unidad de } \\
\text { análisis simple). Estudio exploratorio, descriptivo y explicativo. }\end{array}$ \\
\hline Unidad de análisis & Empresas españolas con procesos de privatización consolidados. \\
\hline $\begin{array}{l}\text { Ámbito } \\
\text { geográfico }\end{array}$ & España. \\
\hline $\begin{array}{l}\text { Universo/ } \\
\text { Población }\end{array}$ & $\begin{array}{l}\text { Empresas privatizadas españolas que forman parte del IGBM y fueron } \\
\text { privatizadas por medio de una OPV en el período 1985-2003. }\end{array}$ \\
\hline Tipo de muestra & $\begin{array}{l}\text { Muestra lógica y teórica (capacidad de generalización analítica del } \\
\text { fenómeno estudiado), no de forma aleatoria (muestreo y generalización } \\
\text { estadística). }\end{array}$ \\
\hline Muestra & Cuatro empresas privatizadas españolas: Endesa, Iberia, Indra y Telefónica. \\
\hline $\begin{array}{l}\text { Métodos de } \\
\text { recogida de la } \\
\text { evidencia }\end{array}$ & $\begin{array}{l}\text { Revisión documental (documentación y archivos). } \\
\text { Cuestionario de preguntas cerradas de elección múltiple. } \\
\text { Realización de entrevistas múltiples en profundidad: abiertas, } \\
\text { semiestructuradas y presenciales. }\end{array}$ \\
\hline $\begin{array}{l}\text { Fuentes de } \\
\text { información }\end{array}$ & $\begin{array}{l}\text { Interna: documentación (memorias, informes y estudios internos), archivos } \\
\text { (páginas web, presentaciones, archivos de imagen y sonido), entrevistas en } \\
\text { profundidad, cuestionarios, contexto físico real. } \\
\text { Externa: publicaciones especializadas, base de datos SABI, informes de } \\
\text { organismos oficiales y medios de comunicación. }\end{array}$ \\
\hline $\begin{array}{l}\text { Informadores } \\
\text { clave }\end{array}$ & $\begin{array}{l}\text { Directivos (mínimo dos) de las empresas de la muestra con participación en } \\
\text { el proceso de privatización. }\end{array}$ \\
\hline $\begin{array}{l}\text { Métodos de } \\
\text { análisis de la } \\
\text { evidencia }\end{array}$ & $\begin{array}{l}\text { Fundamentalmente de tipo cualitativo: } \\
\text { - Descripción individualizada de cada caso } \\
\text { - Búsqueda del grado de coincidencia entre los comportamientos de las } \\
\text { empresas analizadas, con el apoyo de las proposiciones teóricas. } \\
\text { - Creación de explicación teórica (comparación sistemática teórica). }\end{array}$ \\
\hline Enfoque científico & $\begin{array}{l}\text { Inducción analítica a través de la lógica de la réplica (generalización } \\
\text { analítica). Procesos deductivos en la medida que se parte de proposiciones } \\
\text { teóricas de la revisión de teorías. }\end{array}$ \\
\hline $\begin{array}{l}\text { Rigor y calidad } \\
\text { metodológica }\end{array}$ & Validez (constructiva, interna y externa) y fiabilidad. \\
\hline $\begin{array}{l}\text { Fecha de } \\
\text { realización }\end{array}$ & 2005 - enero de 2008. \\
\hline
\end{tabular}

Fuente: Elaboración propia 
Anexo 2: Perfiles básicos de las cuatro empresas analizadas

\begin{tabular}{|c|c|c|c|c|c|c|}
\hline 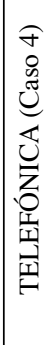 & 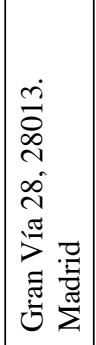 & 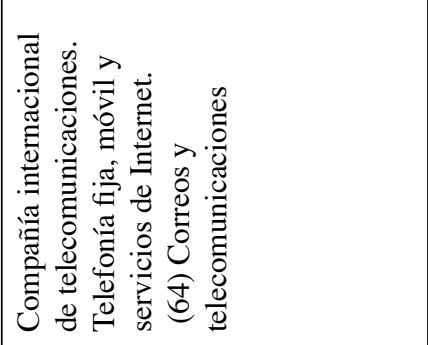 & 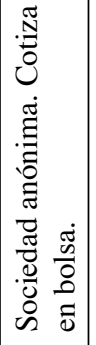 & 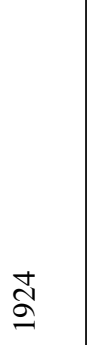 & \begin{tabular}{l}
8 \\
8 \\
0 \\
$\infty$ \\
\multirow{2}{+}{}
\end{tabular} & 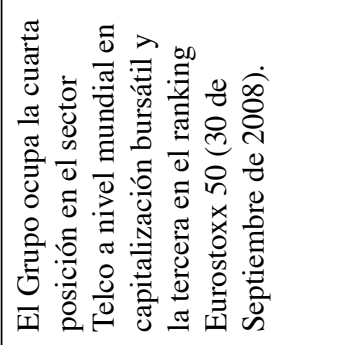 \\
\hline 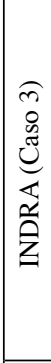 & 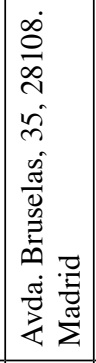 & 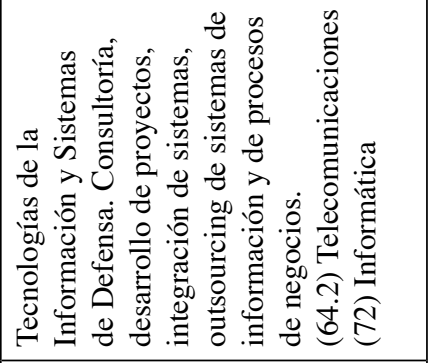 & 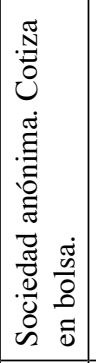 & ๙ & $\begin{array}{l}\text { ô } \\
\text { +े } \\
\text { ते }\end{array}$ & 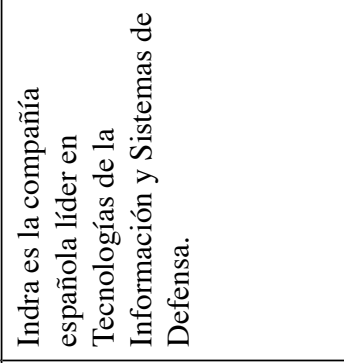 \\
\hline 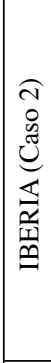 & 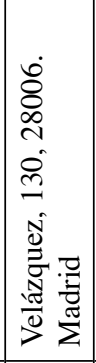 & 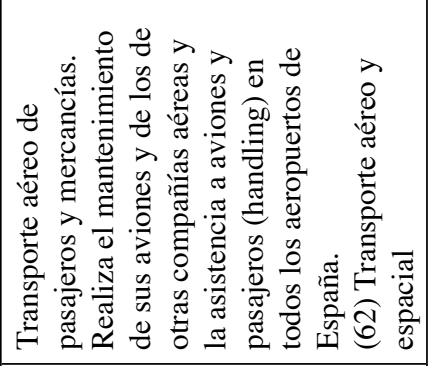 & 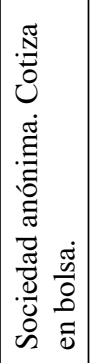 & $\widehat{\widehat{\sigma}}$ & 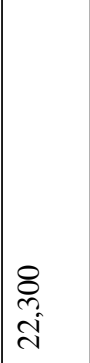 & 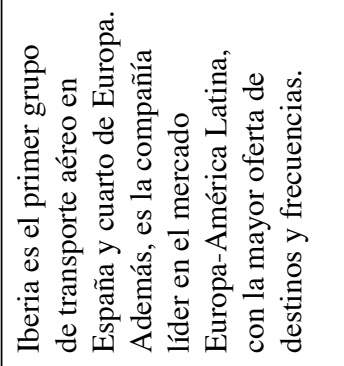 \\
\hline 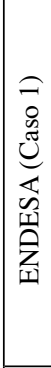 & 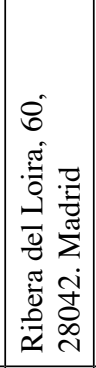 & 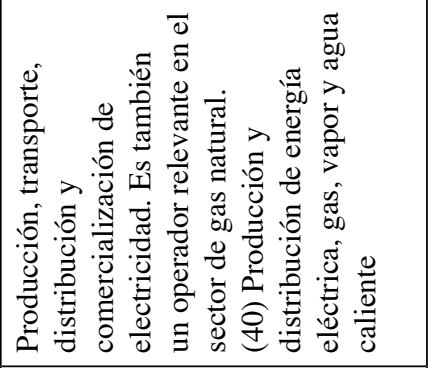 & 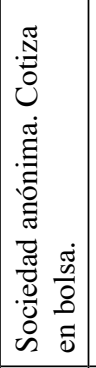 & 导 & $\begin{array}{l}\frac{a}{\partial} \\
\hat{\imath}\end{array}$ & 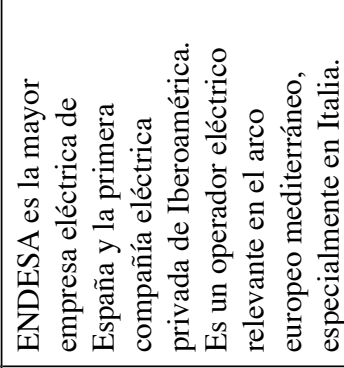 \\
\hline 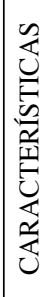 & 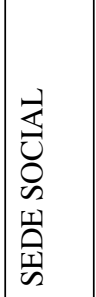 & 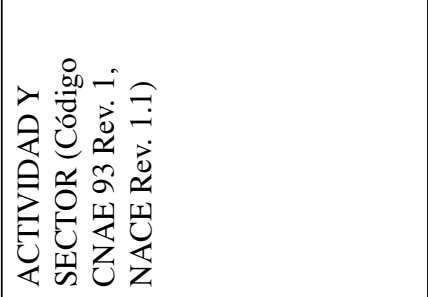 & 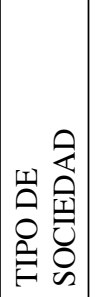 & 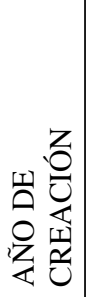 & 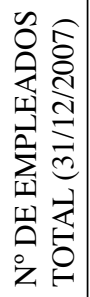 & 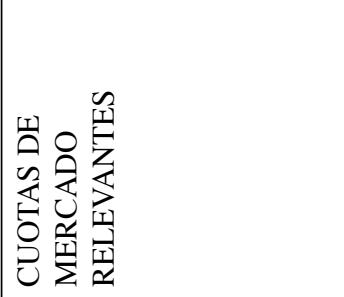 \\
\hline
\end{tabular}




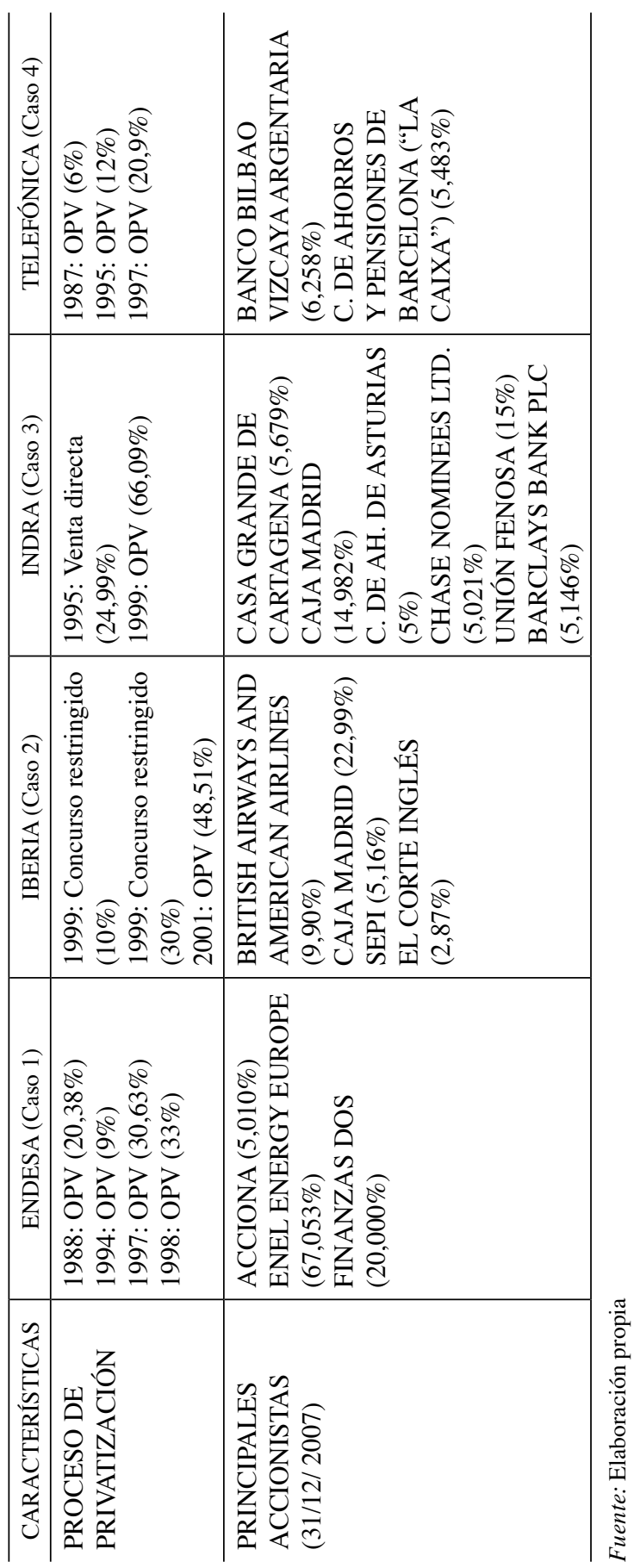




\section{Anexo 3: Pruebas de evaluación del rigor y la calidad de esta investigación}

\begin{tabular}{|c|c|}
\hline Prueba & Táctica \\
\hline $\begin{array}{l}\text { Validez } \\
\text { constructiva }\end{array}$ & $\begin{array}{l}\text { Análisis previo del contexto conceptual y marco teórico (triangulación teórica). } \\
\text { Desarrollo de las proposiciones. } \\
\text { Utilización de distintos métodos de recogida de la evidencia (triangulación meto- } \\
\text { dológica): } \\
\quad>\text { Documentación. } \\
\quad>\text { Realización del cuestionario } \\
\quad>\text { Entrevistas en profundidad. } \\
\quad>\text { Grupo de expertos } \\
\text { Utilización de múltiples fuentes de información (triangulación de datos) para con- } \\
\text { firmación de evidencia en distintas fuentes: } \\
\quad>\text { Interna y externa, directa (primaria) e indirecta (secundaria). } \\
\quad>\text { Tipología diversa: documentación, archivos, entrevistas, cuestionarios, ba- } \\
\quad \text { ses de datos, contexto físico real. } \\
\quad>\text { Diversidad de informadores clave ante mismas cuestiones. } \\
\quad>\text { Evaluación crítica de evidencia comparada según fuentes. } \\
\text { Proceso casi simultáneo y unificado de recogida y análisis de evidencia. } \\
\text { Establecimiento de cadena de evidencia. } \\
\text { Retroalimentación y contacto interactivo con los informadores. } \\
\text { Revisión del informe del caso por parte de informadores clave. } \\
\text { Flexibilidad general e instrumental de la investigación a través de la revisión cícli- } \\
\text { ca del estudio de campo. }\end{array}$ \\
\hline $\begin{array}{l}\text { Validez } \\
\text { interna }\end{array}$ & $\begin{array}{l}\text { Patrón de comportamiento común (apoyo en las proposiciones teóricas). } \\
\text { Creación de explicación (comparación sistemática de la literatura estructurada). }\end{array}$ \\
\hline $\begin{array}{l}\text { Validez } \\
\text { externa }\end{array}$ & $\begin{array}{l}\text { Uso de teorías rivales (triangulación teórica). } \\
\text { Establecimiento de unidad de análisis y selección de casos según el potencial de } \\
\text { información sobre el fenómeno estudiado. } \\
\text { Selección de métodos de recogida de datos (triangulación metodológica) y de } \\
\text { fuentes de información (triangulación de datos) según el potencial de conocimien- } \\
\text { to sobre el fenómeno estudiado. } \\
\text { Aplicación de la lógica replicante (estudio de casos múltiples) para la consecución } \\
\text { de generalización analítica. } \\
\text { Consideración de parte de los resultados de la investigación como hipótesis de } \\
\text { partida de estudios de futuras líneas de investigación. }\end{array}$ \\
\hline Fiabilidad & $\begin{array}{l}\text { Realización de un protocolo del estudio y seguimiento de sus pautas como guía } \\
\text { de acción. } \\
\text { Elaboración de una base de datos que organice, integre y sintetice la información } \\
\text { obtenida de las distintas fuentes de evidencia. }\end{array}$ \\
\hline
\end{tabular}

Fuente: Elaboración propia, basada en Yin $(1994,1998)$ y Villarreal (2007) 


\section{REFERENCIAS BIBLIOGRÁFICAS}

AHARONI, Y. (1981): "Managerial discretion in state owned enterprises" en Vernon, R. y Aharoni, Y. (eds.), State-Owned Enterprise in Western Economies. Londres, Croom Helm, pp. 184-193.

ARCAS, M. J. Y RUIZ, F. J. (1999): "Los efectos de la privatización de empresas sobre su comportamiento económico y financiero: el caso de Repsol”. Hacienda Pública Española, 149, pp. 21-33.

ARIAS, M. (2003): “Metodologías de investigación emergentes en economía de la empresa”, Papers Proceedings 2003, XVII congreso nacional XIII congreso hispano-francés AEDEM, Université Montesquieu Bordeaux IV, Bordeaux, pp. 19-28.

ATKINSON, S.E. AND HALVORSEN, R. (1986): "The relative efficiency of public and private firms in a regulated environment: the case of US electric utilities", Journal of Public Economics, 29, pp. 281-294.

BOARDMAN, A.E.; LAURIN, C. AND VINING, A.R. (2003): "Privatization in North America", in David Parker and David Saal (eds.) International Handbook on Privatisation, Edward Elgar, Cheltenham, U.K., pp. 129-160.

BOUBAKRI, N. AND COSSET, J.C. (1998): “The financial and operating performance of newly privatised firms: evidence from developing countries", Journal of Finance, 53(3), pp. 1081-1110.

BOUBAKRI, N. AND HAMZA, O. (2007): "The dynamics of privatization, the legal environment and stock market development", International Review of Financial Analysis, 16(4), pp. 304-331.

BOUBAKRI, N.; COSSET, J.C. AND GUEDHAMI, O. (2005): "Liberalization, corporate governance and the performance of privatized firms in developing countries", Journal of Corporate Finance, 11, pp. 767-790.

BOYCKO, M.; SHLEIFER, A. AND VISHNY, R. (1996): "A theory of privatization”, Economic Journal, 106, pp. 309-319.

BRADLEY, M.; JARREL, G. Y KIM, E. H. (1984): "On the existence of an optimal capital structure: Theory and evidence". Journal of Finance, vol. 39, pp. 857-878.

BREALEY, R. A. Y MYERS, S. C. (1995): Fundamentos de financiación empresarial. McGrawHill, Madrid.

BRUGGINK, T.H. (1982): "Public versus regulated private enterprise in the municipal water industry: a comparison of operating costs", Quarterly Review of Economics and Business, 22(1), pp. 111-125.

BURNS, P. AND WEYMAN-JONES, T. (1994): Regulatory incentives, privatisation and productivity growth in UK electricity distribution, CRI Technical Paper 1, Centre for the Study of Regulated Industries, London, Chartered Institute of Public Finance and Accountancy.

CHIVA, R. (2001): "El estudio de casos explicativo. Una reflexión". Revista de Economía y Empresa, vol. XV, no 41, pp. 119-132.

CUERVO, A. (2004): "Empresa pública y privatización”, Papeles de Economía Española, 100, pp. $147-160$.

D’SOUZA, J. AND MEGGINSON, W.L. (1999): "The financial and operating performance of newly privatized firms during the 1990s", Journal of Finance, 54(4), pp. 1397-1438.

D’SOUZA, J.; MEGGINSON, W.L. AND NASH, R. (2005): "Effects of institutional and firm specific characteristics on post-privatization performance: Evidence from developed countries", Journal of Corporate Finance, 11(5), pp. 747-766.

DEWENTER, K. AND MALATESTA, P.H. (2000): "State-owned and privately-owned firms: an empirical analysis of profitability, leverage and labour intensity", American Economic Review, 91(1), pp. 320-334. 
DI LORENZO, T.J. AND ROBINSON, R. (1982): "Managerial objectives subject to political market constraints: electric utilities in the US", Quarterly Review of Economics and Business, 22(2), pp. 113-125.

DURÁN, J. J. (2004): "Las empresas multinacionales privatizadas. Especial referencia al caso español" en VV. AA., Teoría y política de privatizaciones: su contribución a la modernización económica. Análisis del caso español, Fundación SEPI, Ediciones Cinca, Madrid, pp. 833-855.

ECKEL, C.; ECKEL, D. AND SINGAL, V. (1997): "Privatization and efficiency: Industry effects of the sale of British Airways", Journal of Financial Economics, 43, 275-298.

EISENHARDT, K.M. (1989): "Building theories from case study research", Academy of Management Review, 14, pp. 532-550.

EISENHARDT, K.M. (1991): "Better Stories and Better Constructs: The Case for Rigor and Comparative Logic", Academy of Management Review, 16(3), pp. 620-627.

EISENHARDT K.M. AND GRAEBNER M.E. (2007): "Theory building from cases: opportunities and challenges", Academy of Management Journal, 50(1), pp. 25-32.

ESTRIN, S. (2002): "Competition and corporate governance in transition", Journal of Economics Perspectives, 16(1), pp. 101-124.

FOREMAN-PECK, J. (1989): Ownership, competition and productivity growth: the impact of liberalisation and privatisation upon British Telecom. Warwick Economic Research Papers Series (TWERPS), $\mathrm{n}^{\circ} 338$, University of Warwick.

FOREMAN-PECK, J. AND WATERSON, M. (1985): "The comparative efficiency of public and private enterprise in Britain: electricity generation between the world wars", The Economic Journal, 95, pp. 83-95.

GARCÍA, M.T. Y MARIZ, R.M. (2003): El efecto de las privatizaciones sobre la gestión empresarial. AEDEM, XVII Congreso Nacional y XIII Congreso Hispano-Francés, Bordeaux, pp. 1087-1096.

GIBBERT, M.; RUIGROK, W. AND WICKI, B. (2008): "What passes as a rigorous case study?", Strategic Management Journal, 29, pp. 1465-1474.

GÓMEZ, M. Y GARCÍA, A. (2004): "La privatización y el aumento de competitividad de las empresas españolas", in VV. AA., Teoría y política de privatisaciones: su contribución a la moderniiación económica. Análisis del caso español, Fundación SEPI, Ediciones Cinca, Madrid, pp. 857-877.

GONZÁLEZ-PÁRAMO, J. M. Y HERNÁNDEZ DE COS, P. (2004): “La evolución económicofinanciera de las empresas privatizadas, 1983-1996”, en VV. AA., Teoría y política de privatizaciones: su contribución a la modernización económica. Análisis del caso español, Fundación SEPI, Ediciones Cinca, Madrid, pp. 655-670.

IRANZO, J.E. (2004): "De la empresa estatal a la multinacional: el papel de las antiguas empresas de propiedad pública en la expansión de la economía española” in VV. AA., Teoría y política de privatizaciones: su contribución a la modernización económica. Análisis del caso español, Fundación SEPI, Ediciones Cinca, Madrid, pp. 813-832.

LAFFONT, J. J. Y TIROLE, J. (1993): A theory of incentives in procurement and regulation. MIT Press, Cambridge, Massachusetts.

LAURIN, C. AND BOZEC, Y. (2001): "Privatization and productivity improvement: the case of Canadian National", Transportation Research Part E: Logistics and Transportation Review, 37(5), pp. 355-374.

LYNK, E.L. (1993): "Privatisation, joint production and the comparative efficiencies of private and public ownership: the UK water industry case", Fiscal Studies, 14(2), pp. 98-116.

MARTIN, S. AND PARKER, D. (1995): "Privatization and economic performance throughout the UK business cycle", Managerial and Decision Economics, 16, pp. 225-237. 
MEGGINSON, W. L. (2004): "Los efectos macroeconómicos de la privatización", en VV. AA., Teoría y política de privatizaciones: su contribución a la modernización económica. Análisis del caso español, SEPI, Ed. Cinca, Madrid, pp. 391-444.

MEGGINSON, W.L.; NASH, R.C. AND RANDENBORGH, M.VAN. (1994): "The financial and operating performance of newly privatized firms: an international empirical analysis", Journal of Finance, 49(2), pp. 403-452.

MEGGINSON, W.L.; NASH, R.C.; NETTER, J. AND POULSEN, A. (2004): "The choice of private versus public capital markets: evidence from privatizations", Journal of Finance, 59(6), pp. 2835-2870.

MILLWARD, R.E. AND PARKER, D.M. (1983): "Public and Private Enterprise: Comparative Behaviour and Relative Efficiency" in Robert Millward, Parker, Rosenthal, Sumner and Topham (eds.) Public Sector Economics. Longman, New York, pp. 199-274.

MILLWARD, R. AND WARD, R. (1987): “The costs of public and private gas enterprises in late $19^{\text {th }}$ century Britain", Oxford Economic Papers, 39, pp. 719-737.

PARKER, D. AND KIRKPATRICK, C. (2005): "Privatisation in developing countries: A review of the evidence and the policy lessons", Journal of Development Studies, 41(4), pp. 513-541.

PRYKE, R. (1982): "The comparative performance of public and private enterprise”, Fiscal Studies, 3(2), pp. 68-81.

RONDINELLI, D.A. (2004): "Políticas e instituciones para dirigir el proceso de privatización: la experiencia internacional" in VV. AA., Teoría y política de privatizaciones: su contribución a la modernización económica. Análisis del caso español, Fundación SEPI, Ediciones Cinca, Madrid, pp. 115-141.

SHLEIFER, A. AND VISHNY, R. (1994): "Politicians and firms". Quarterly Journal of Economics, vol. 109, no 4, pp. 995-1025.

SIGGELKOW, N. (2007): "Persuasion with case studies", Academy of Management Journal, 50(1), pp. 20-24.

SOSA, S. (2006): La génesis y el desarrollo del cambio estratégico: un enfoque dinámico basado en el momentum organizativo. Tesis doctoral, U. Las Palmas de G. Canaria.

TIAN, G.L. (2000): State shareholding and corporate performance: a study of a unique Chinese data set, Working Paper, London Business School.

VILLARREAL, O. (2007): La Estrategia de Internacionalización de la Empresa. Un Estudio de Casos de Multinacionales Vascas, Tesis Doctoral, Universidad del País Vasco-Euskal Herriko Unibertsitatea, Bilbao.

YIN, R. K. (1989): Case study research: design an methods. Sage Publications, London.

YIN, R.K. (1993): Applications of case study research. Newbury Park, Sage Publications, London.

YIN, R.K. (1994): Case study research. Design and methods (2 ${ }^{\mathrm{a}}$ ed.). Newbury Park, Sage Publications, London.

YIN, R.K. (1998): “The Abridged Version of Case Study Research", in Bickman, L. and Rog, D. J. (eds.): Handbook of Applied Social Research Methods, Sage Publications, Thousand Oaks, pp. 229-259.

ZAHRA, S.A.; IRELAND, R.D.; GUTIERREZ, I. AND HITT, M.A. (2000): "Privatization and entrepreneurial transformation: emerging issues and a future research agenda", Academy of Management Review, 25(3), pp. 509-524. 
